\title{
Die Theaterszene des Rosswalder Schlosses zwischen 1750 und 1760 und ihre literaturhistorischen Metamorphosen
}

\author{
Iveta ZLÁ
}

\begin{abstract}
Abstrakt
The theater scene at Rosswald Castle in the 1750s and 1760s and its metamorphoses in literary history

The article is about mediating an involvement in the development of the Rosswald Castle Theater between 1750 and 1760. Attention is paid to the theater repertoire and the representation of the artistic atmosphere in the Rosswald Castle. Last but not least, the article outlines the role of the Rosswald sentimental garden, in which the dramas, operas and ballet performances were also located. The article points to the incorporation of creative impulses of Enlightenment into the repertoire of the Rosswald cultural scene. The focus of the investigation is on the activity of Johann Heinrich Friedrich Müller, who implemented the dramatic rules of the Enlightenment in the Rosswald Castle Theater.
\end{abstract}

Keywords: Rosswald Castle, Baroque, Enlightenment, Drama, Ballet, Opera, Johann Heinrich Friedrich Müller, Count Albert Joseph Hoditz, Rosswald Castle Garden, Castle Theater.

DOI: doi.org/10.15452/StudiaGermanistica.2021.28.0008

\section{Einleitung in die Thematik}

Die Zeit zwischen Barock und Aufklärung war in den Böhmischen Ländern mit einer florierenden Entwicklung des künstlerischen Lebens verbunden. Aufmerksamkeit verdienen u. a. künstlerische Aktivitäten des Adels, die einerseits auf den Ansprüchen einer angemessenen barocken Repräsentation fußen, andererseits mit intensiven Bemühungen um die Verbreitung der Literatur, Kultur und Bildung verbunden waren. Diese Ambitionen sind nicht nur in Böhmen präsent, sondern rangen in dieser Schwellenzeit auch in Mähren und Schlesien empor. Stellvertretend ist das künstlerische Engagement des Grafen Jan Rottal (1605-1674) sowie seines Nachfolgers Franz Karl Rottal (1690-1672) zu nennen, das mit dem Schlosstheater im mährischen Holleschau ${ }^{1}$ zusammenhing. Das Schloss des Grafen Graf Dominik Ignaz Chorinsky (1729-1792) im westschlesischen Groß Hoschütz ${ }^{2}$ war

Holešov

2 Velké Hoštice 
vor allem im letzten Viertel des 18. Jh. mit dem regen kulturellen Leben verknüpft. Einen großen kulturellen Aufschwung haben ebenfalls die Schlösser in Rosswald $^{3}$, Gotschdorf ${ }^{4}$, Geppersdorf ${ }^{5}$ und Johannesberg in Jauernig ${ }^{6}$ erlebt. Diese Tatsache hing mit dem kulturellen Engagement des Adels sowie der in Jauernig tätigen Breslauer Bischöfe zusammen. Einen entscheidenden Anteil an diesen westschlesischen kulturellen Highlights hatten besonders die Grafen Albert Joseph Hoditz (1706-1778) und Dominik Chorinsky (1729-1792) sowie der Bischof Philipp Gotthard Schafgotsch (1715-1795). Obwohl das kulturelle Bild aller genannten Schlösser zahlreiche Forschungsimpulse bietet, bildet den Schwerpunkt der vorliegenden Studie die Rosswalder Schlossbühne zwischen 1750 und 1760 .

Der Graf Albert Joseph Hoditz hat sein Rosswalder Schloss ungefähr seit 1741 allmählig zum „Ort der Museen und Grazien“ verwandelt, dessen Parkanlagen durch zahlreiche Seen, Wasserfälle und Grotten geschmückt wurden. Die Atmosphäre dieser prächtigen Gartenanlagen wurde durch die Statuen mitgeprägt, die an die antike Kunst erinnerten. Kein Wunder, dass in die Rosswalder Gartenlandschaft oft die Dramen- und Opernaufführungen situiert wurden. Obwohl in Schlesien zwischen 1756 und 1763 der Siebenjährige Krieg herrschte, wurde das Rosswalder Herrschaftsgut zu einer „Oase“ des Friedens und künstlerischer Inspiration. In diesem kulturellen Panorama blühte auch das Roswalder Schlosstheater, in dessen Repertoire besonders zwischen 1750 und 1760 die neuesten literaturhistorischen Tendenzen Resonanz gefunden haben.

In diesem Zusammenhang gehört zu den Grundintentionen dieser Studie, eine Einsicht ins Repertoire des Rosswalder Theaters zwischen 1750 und $1760 \mathrm{zu}$ bieten und seine dramentheoretischen Impulse anzudeuten. Methodologisch geht die Untersuchung von den Prinzipien der hermeneutischen Forschung aus, verdeutlicht die kulturhistorischen Hintergründe und zielt auf die Darstellung eines plastischen Bildes der Rosswalder Kulturszene ab.

\title{
2. Die Rosswalder Theaterbühne: Forschungsausgangspunkte
}

Informationen über das Rosswalder Theater bietet das archivalische Fond des Großgrundbesitzes Groß Hoschütz, in dem neben den Sentenzen des Grafen Hoditz das Vorspiel, Die Vereinigung des Trauerspiels, des Lustspiels, der Tonkunst und des Tanzes‘ (ZAO, FVH:163/93) Johann Heinrich Friedrich Müllers zu finden ist (Vgl. Zlá 2019:87-99). Einzelheiten über die kulturelle Atmosphäre im Rosswalder Schloss sind ebenfalls in der Reisebeschreibung, Schattenriss der Annehmlichkeiten von Roswalde des Breslauer Arztes Balthasar Ludwig Tralles nachzulesen, der Rosswald 1756 besucht hat.

Das Theatergeschehen in der Rosswalder Schlossresidenz ist ebenfalls vor dem Hintergrund der hiesigen Musikbühne zu betrachten, ${ }^{7}$ die von der Tätigkeit des Komponisten Karl Ditters von Dittersdorf (1739-1799), des italienischen Librettisten Ignaz Pintus (1714-1786), des Freunds Ludwig van Beethovens Karl Hanke, ${ }^{8}$ des Schülers Christoph Willibald Glucks Heinrich Klein ${ }^{9}$ etc. beeinflusst war.

Den Kunstgeschmack des Grafen Hoditz hat zweifelsohne seine Lektüre beeinflusst, die das Inventar der Rosswalder Schlossbibliothek andeuten könnte. In der Bibliothek lagen die Kompendien aus den Bereichen wie Geographie, Jura, Chemie, Alchimie, Gartenkunst usw. Unter den literarischen Werken dominierten die die Komödien, Tragödien und Possen von Molière (1622-1673), Pierre

\author{
Slezské Rudoltice \\ Hošt'álkovy \\ Linhartovy \\ Jánský Vrch v Javorníku \\ Milan Myška weist auf die Tätigkeit einiger Musiker im Rosswalder Schlosstheater sowohl unter Karl Joseph Hoditz als \\ auch unter seinem Sohn, Albert Joseph Hoditz hin (Myška 2011:148-164). \\ Die Lebensdaten sind unbekannt. \\ Die Lebensdaten sind unbekannt.
}


Corneille (1606-1684), François Marie Arouet Voltaire (1694-1778), Ludwig Holberg (1684-1754) sowie die Libretti von Ignaz Pintus (1714-1786). Den Einblick in die neueste deutsche Literatur repräsentierten dann die Dramen Gotthold Ephraim Lessings (1729-1781) sowie die literarisch ausgerichteten Periodika. (Vgl. Myška 2011:148-164)

Obwohl sich das Repertoire des Rosswalder Schlosstheaters wahrscheinlich durch eine schöpferische Vielfalt auszeichnete, sind die Informationen über die Theateraufführungen in diesem aristokratischen Sitz spärlich. In der einschlägigen Forschungsliteratur tauchen Angaben über Aufführungen der Werke der italienischen, französischen, dänisch-norwegischen und deutschen Provenienz auf, was ebenfalls der Einblick in den Buchbestand der Schlossbibliothek belegt (Myška 2011:117-121). In diesem Zusammenhang wurden in Rosswald sowohl die mit der Literaturepoche der Aufklärung verknüpften Tragödien Voltairs Zaire‘ und ,Alzire, ou les Americains', als auch klassizistische Dramen Molières (1622-1673) aufgeführt. (Vgl. Myška 2011:117-121)

In die Geschichte des Rosswalder Schlosstheaters sind auch Aufführungen der dramatischen Werke des Grafen Albert Joseph Hoditz eingegangen. Er verfasste die Libretti zu Opern, Die vergötterte Sophia' und, Einquartierung der Panduren' und in sein literarisches Schaffen sind auch die Possen ,Doctor und Apotheker' und ,Der theuere Ziegenbock' eigegangen. Diese Libretti und Possen sind jedoch leider verschollen.

Neben dem Einblick des Grafen Albert Joseph Hoditz in die zeitgenössische europäische Literatur, der Ausbildung seiner Diener zu Schauspielern und Opernsängern (vgl. Myška 2011:164-201) und seiner Zusammenarbeit mit einigen westschlesischen Schlossresidenzen (Zlá 2020:63-71) war im Rosswalder kulturellen Leben die Tätigkeit des Dramatikers und Schauspielers Johann Heinrich Friedrich Müller (1738-1815) von entscheidender Bedeutung

\section{Augen und Ohren gleichsam [...] ergreift bewundernswürdige Wollust. Das Rosswalder Schlosstheater im Fokus der Reisebeschreibung von Baltha- sar Ludwig Tralles (1708-1797), Schattenriss der Annehmlichkeiten von Roswalde}

Das Rosswalder Schloss lockte in diese Kulturstätte viele Künstler, führende Persönlichkeiten des zeitgenössischen gesellschaftlich-politischen Lebens und nicht zuletzt Reisende. Das Bild dieser Schlossresidenz ist in die Reisebeschreibung von Balthasar Ludwig Tralles, Schattenriss der Annehmlichkeiten von Roswalde“ (Tralles 1776) eingegangen, die 1776 erschienen ist. Der Reisebericht thematisierte die von Tralles 1756 unternommene Reise nach Rosswald und seinen Aufenthalt in dieser Schlossresidenz.

Einzelheiten über das Schlosstheater sind vor allem dem Kapitel, Die Oper' zu entnehmen. Tralles schätzt den Gesang der Opernsänger hoch, was diese Worte belegen: Augen und Ohren gleichsam als nach einem magischen Gesang, ergreift bewundernswürdige Wollust (Tralles 1776:79). Dennoch verdeutlicht die Reisebeschreibung, dass als Vorspiel auf dem Spielplan auch die Aufführungen standen, die die Züge der sog. Hanswurstiaden trugen. In ihrem Mittelpunkt stand die Figur Pancraz, die mit marktschreierischer Geschwätzigkeit und Burlesken-Anstand das marmorne Herz seiner angebeteten Isabel zu beugen und zu weichen sucht [e] (Tralles 1776:81).

Obwohl die kulturelle Atmosphäre und das künstlerische Niveau der Rosswalder Aufführungen von Tralles meistens positiv bewertet wurden, tauchen im Reisebericht auch kritische Bemerkungen seines Autors auf. Tralles kritisiert die Unnatürlichkeit und Regellosigkeit der aufgeführten Dramen. Diese Merkmale wurden durch die Hervorhebung des Phantastischen bedingt, das noch vor dem Anfang der dramatischen Tätigkeit Johann Heinrich Friedrich Müllers im Rosswalder Schlosstheater akzentuiert wurde (Vgl. Tralles 1776:81). Der in der Reisebeschreibung vermittelte Einblick ins Theaterleben dieser Schlossresidenz belegt die Anlehnung dieser Theaterszene an die Prinzipien des Barocktheaters. Die Schauspiele wurden mit dem Tanz und Gesang zu einem organischen Komplex verbunden. Das Phantastische war oft mit Vergänglichkeit verbunden, die meistens jedoch nicht als 
Gegenpol des Daseins betrachtet wurde. Die Eitelkeit wurde als untrennbarer Teil der menschlichen Existenz wahrgenommen.

Im Rosswalder Theater wurden nicht zuletzt die Dramen von Hoditz aufgeführt und seine Gedichte vorgelesen. Da das literarische Schaffen des Grafen Albert Joseph Hoditz vorwiegend verschollen ist, gewinnen die durch die Reisebeschreibung vermittelten Informationen über das Werk dieses Adligen an Bedeutung. Tralles vergleicht die Gedichte von Hoditz mit der Poesie des Schweizer Autors Albrecht Haller (1708-1777). Die überlieferten Gedichte des Grafen Hoditz trugen meistens - ebenso wie das Werk Albrecht Hallers - lehrhafte Züge, wodurch sie bereits zur Aufklärung inklinierten.

Im Reisebericht von Tralles wird die Handlungslinie einer unbetitelten Komödie nachgezeichnet, die der Erzähler als ein sehr artiges und dem Geist unserer Zeit angemeßenes Schauspiel (Tralles 1776:126) charakterisiert. Vor ihrem Hintergrund wird die Nachahmung der Wirklichkeit in den Vordergrund gerückt.

In die Reisebeschreibung sind einige von Tralles vermittelte Gedichte eingegangen, die während des Schäferfestes vorgetragen wurden und mit ausgewählten Figuren der antiken Mythologie verbunden waren. Unter diesen Figuren dominierte der Gott Arkadiens Pan, der sowohl in der antiken Mythologie, als auch in diesen Schäferspielen die Rolle des Hirtenbeschützers übernommen hat. Für Pales wurde das folgende Gedicht vorgetragen, in dessen Mittelpunkt die Nymphen und Najaden standen:

Sieh, es bringen die Nymphen

in vollen

Körbgen Lilien dir, dir pflückt die

Weiße Najade

Bleiche Violen und hohen Mohn, und

Bindet Narcissen

Und süß düftende Blüthen von Dill dazu. (Tralles 1776:165)

Die Verbindung der bukolisch geprägten, reimlosen Dichtung mit der Rosswalder Gartenszenerie rückt die Natur in den Vordergrund und räsoniert mit einer der tragenden Ideen der Aufklärung, die die Annäherung an die Natur durchsetzt. Die im Gedicht erfassten Nymphen und Najaden verkörpern die Naturkräfte und ihre Macht (Vgl. Tralles 1776:165). Die als Anadiplose stilisierte Wiederholung des Personalpronomens „dir“" wird durch die Begrüßung der Göttin Pales Athena und ihre Beschenkung mit Blumen intensiviert.

Da die Schäferdichtung im Rosswalder Garten vorgetragen wurde, wendet sich der Erzähler der Skizzierung dieser Gartenlandschaft zu. Er weist implizit auf die Verbindung der Natur mit Welt der Literatur und Musik hin, die jedoch in das Reich der Bildhauerkunst und Gartenarchitektur eingebettet werden.

Obwohl in den dargelegten Tagebuchaufzeichnungen über das Rosswalder Schlosstheater bereits Anzeichen von der sich allmählich durchsetzenden Aufklärung zu finden sind, überwogen in den 1750er Jahren im Rosswalder Schlosstheater noch barocke Motive und Regeln, die sich u. a. in Verbindung des Musikalischen und Dramatischen mit dem Tanz auszeichneten.

\section{Johann Heinrich Friedrich Müllers kulturelles Engagement in Rosswald: Durchsetzung des aufklärerischen Theaters}

Johann Heinrich Friedrich Müller kam 1757 nach Rosswald (vgl. Zlá 2019:169). Er war wahrscheinlich seit dem Anfang der 1750er Jahre in der Theatertruppe Johann Friedrich Schönemanns (1704-1782) tätig, die jedoch mit finanziellen Problemen kämpfte. Auf Empfehlung des Freiherrn Wilhelm Raimund Wittorf ${ }^{10}$ kam er an den Rosswalder Hof, an dem er mit diversen Pflichten be-

10 Der Freiherr Wilhelm Raimund Wittrof wurde nach 1720 geboren. Seine genauen Lebensdaten sind jedoch unbekannt. Vgl. URL 1. 
auftragt wurde. Er hat in der Rosswalder Schlossschule beispielsweise Geographie und Heraldik unterrichtet und war Sekretär des Grafen Albert Joseph Hoditz. Darüber hinaus gehörte zu seinen Aufgaben die Ausbildung der Schauspieler, die im Rosswalder Schlosstheater tätig waren. Unter seinem Einfluss wurden einige Persönlichkeiten ausgebildet, die später in den renommierten Theatern wie z. B. in Wien, Breslau, Hamburg usw. emporragten. ${ }^{11}$ (vgl. Myška 2011:166-173) Da er nach Rosswald schon mit bestimmten Theatererfahrungen und einem Einblick in die zeitgenössischen Tendenzen im literarischen Bereich kam, konnte er seine Kenntnisse während seines Rosswalder Engagements gut verwerten.

Im Rosswalder Theater wurden unter seiner Leitung in der Zeitspanne 1758-1760 einige Komödien Molières einstudiert sowie die Dramen ,El malgastodor' und ,Les glorieux“ Nèricault Destoucheses (vgl. Zlá 2019:169) aufgeführt. In den Intentionen der europäischen Aufklärung standen die unter der Leitung Müllers gespielten Dramen ,Miss Sara Sampson' Gotthold Ephraim Lessingssowie ,Alzire‘, ,Zaire‘ und ,Oedipe‘ François Marie Arouet Voltaires und ,George von Barnwel, oder der Kaufmann von London' George Lilos (vgl. Müller 1802:33). Müllers Rosswalder künstlerische Laufbahn stellt ein Prolog zu seiner späteren Tätigkeit auf der Wiener Hof- und Nationalschaubühne dar, die in seinen autobiographischen Werken ,Geschichte und Tagebuch der Wiener Schaubühne“ (Müller 1771) und ,J. H. F. Müllers Abschied von der k. k. Hof- und National-Schaubühne' (Müller 1802) vorgestellt wurde.

Obwohl Müller für die Rosswalder Theaterbühne einige klassizistische Dramen Molières einstudiert hat, hat er sich bereits Ende der 1750er Jahre, d. h. lediglich fünf Jahre nach der Prämiere dieses Dramas, um die Aufführung des Trauerspiels ,Miss Sara Sampson“ (Lessing 1755) im Rosswalder Theater verdient gemacht (Myška 2011:169). Durch die Aufführung dieses Trauerspiels wurden die Zuschauer auf der Rosswalder Theaterbühne mit den moralischen und familiären Werten sowie Gefühlen konfrontiert. Im Mittelpunkt der Dramenhandlung stand nicht mehr der Adel, sondern die Vertreter des Bürgertums. In diesem Zusammenhang wurden die Prinzipien der sog. Ständeklausel völlig abgeschafft und der Dramenablauf beruhte auf der Theorie der gemischten Charaktere. Diese dramentheoretischen Schwerpunkte legt Müller auch in seinem Vorspiel ,Vereinigung des Trauerspiels, des Lustspiels, der Tonkunst und des Tanzes‘ (ZAO, FVH 163/93) dar. Eine der zentralen Intentionen des Vorspiels Müllers hat die Unterhaltung der Zuschauer dargestellt, während das Trauerspiel Lessings auf die Belehrung und Erziehung des Publikums abzielte. Im Trauerspiel, Miss Sara Sampson“ sind nicht zuletzt die Grundideen der Empfindsamkeit zu finden, in der das Privatglück in den Vordergrund gerückt wurde.

Die dramatische Tätigkeit Müllers hat im Rosswalder Schlosstheater einen Wendepunkt markiert, der mit der Durchsetzung der Prinzipien des aufklärerischen Dramas zusammenhängt. Diese Tatsache hat er bereits im Vorwort zu seinem Vorspiel, Vereinigung des Trauerspiels, des Lustspiels, der Tonkunst und des Tanzes angedeutet.

Dieses Bühnenstück wurde als Vorspiel zum Drama François Marie Arouet Voltaires ,Alzire ou les Americains' vorgesehen, was die folgenden Worte belegen: Es wird nach diesem Vorspiele [...] Alzyre [François Marie Arouet Voltaires], welches in Leipzig ins Deutsche übersetzt worden ist, auf unserer Schaubühne vorgestellet werden (ZAO, FVH 163/93).

Müller formuliert bereits im Vorwort zu diesem Drama die Prinzipien, die für die dramatische Darstellung der literarischen Vorlage richtungsweisend sind. Seiner Meinung nach ist jede Handlungslinie im Kontext des ganzen Dramas zu betrachten. Dementsprechend sind auch die schauspielerischen Leistungen an den Gesamtkomplex des Dramas gebunden. In den Vordergrund der Handlung werden die moralische Verbesserung sowie die Erweckung der Leidenschaften gerückt, was das folgende Zitat andeutet:

Wollen Sie aber Leidenschaften und besonders Mitleiden erregen, so müssen Sie sich Hochachtung gegen die jenigen Personen erwecken die Sie vorstellen. [...] Denn es braucht nicht viel Kunst, Sa-

11 Es handelte sich z. B. um Anna Maria Stormke (Lebensdaten unbekannt), Elsa Springer (Lebensdaten unbekannt), Anna Maria Theyner (Lebensdaten unbekannt) etc. (vgl. Myška 2011:174-175). 
chen zu sagen, die man nicht denket, aber in der Art sich auszudrücken, verrät sich die Art zu denken. (ZAO, FVH 163/93)

Dabei werden Furcht und Leid akzentuiert, die in die Katharsis münden. Katharsis trägt zur Verwandlung des menschlichen Verhaltens bei und erweckt positive Fertigkeiten. Diese didaktisch untermauerte Verwandlung basiert auf der Identifikation mit handelnden Figuren. Ihr Ziel stellt die moralische Verbesserung dar.

Müllers dramatische Reflexionen umkreisen die zentralen Gedanken der Aufklärung. Trotzdem sind in seinen Ausführungen innovative dramentheoretische Überlegungen zu finden. Obwohl Lessings dramentheoretische Prinzipien bereits zum Fundament seiner Lustspiele ,Der Freigeist", ,Die Juden“ von 1749 sowie in seinem Trauerspiel ,Miss Sara Sampson' von 1755 wurden, hat er diese Überlegungen zwischen 1767-1769 in seiner Schrift ,Hamburgische Dramaturgie‘ explizit zum Ausdruck gebracht. Die ,Hamburgische Dramaturgie“ wurde jedoch erst elf Jahre nach dem Vorspiel ,Vereinigung des Trauerspiels, des Lustspiels, der Tonkunst und des Tanzes` Johann Heinrich Friedrich Müllers beendet, dessen Vorrede 1758 gehalten wurde.

Das Vorspiel Müllers ,Vereinigung des Trauerspiels, des Lustspiels, der Tonkunst und des Tanzes' kündigt eine abwechslungsreiche Verbindung des Lustspiels und Trauerspiels mit Musik und Tanz an, die die visuellen sowie akustischen Wahrnehmungen einbeziehen. Im Mittelpunkt der Handlung stehen die Personifikationen des Lustspiels, Trauerspiels, der Tonkunst und des Tanzes, die neben der Freude und Faulheit als zentrale Figuren betrachtet werden. Sie kommen abwechselnd in fünf Auftritten auf Bühne. Ihre Darstellung wird durch den Monolog Apollons, des Königs aller Künste, vollbracht.

In der Ansprache Müllers an die Schauspieler sind ebenfalls Intentionen zu finden, die auf die Tragödie Voltairs ,Alziere‘ eingehen. Im Drama werden religiöse Werte hervorgehoben, die mit moralischen Werten insgesamt in Zusammenhang gesetzt werden:

seine Liebe [die Liebe von Zamore] bekommt einen merklichen Zusatz, wenn man erwägt, daß er das Heydenthum verlassen, aus einem höhern Triebe, mit einer brennenden Begierde, das Christentum ergriffen hat. (ZAO, FVH 163/93)

Im Vorspiel wird die Theorie der sog. gemischten Charaktere umgesetzt. Durch die Auffassung wird negative Handlung der zentralen Dramenfiguren durch ihre positiven Taten überwunden, was die Rührung der Zuschauer zur Folge hat. Zur Typisierung neigt lediglich die Figur von Alvares, die jedoch durch ihre positiven Eigenschaften die Erziehung des Publikums unterstützt. Die Rührung der Zuschauer erfolgt durch ihre Identifikation mit Dramenfiguren. Dennoch werden in diesem Drama immer noch die Regeln der sog. Ständeklausel beachtet, denn seine Handlung wird in die ausschließlich adelige Gesellschaft eingebettet. Im literaturhistorischen Fokus sind jedoch mangelnde Informationen über die schauspielerische Besetzung und über die Leistungen der Schauspieler als defizitär zu betrachten. Der Vorrede ist lediglich die lobende Einstellung Müllers zur schauspielerischen Leistung von Mariana Justin zu entnehmen. (Vgl. ZAO, FVH, 163/93)

Die Vorrede Müllers bietet jedoch auch einen Einblick ins Theaterprogramm von 1758. Auf die Aufführung des Vorspiels, Vereinigung des Trauerspiels, des Lustspiels, der Tonkunst und des Tanzes` folgten die Ballettvorstellung ,Der verjüngte Greis' und ein Nachspiel (vgl. ZAO, FVH, 163/93). Obwohl die Informationen über die Ballettauführung spärlich sind, handelte sich wahrscheinlich um das Werk Johannes Jungheims (1698-1767). Diese Tatsache deutet der im Staatsarchiv Ulm befindliche Theaterzettel an, ${ }^{12}$ laut dem die Ballettvorstellung ,Der verjüngte Greis ebenfalls unter der Leitung des Schauspielers und Dramatikers Karl von Morocz aufgeführt wurde. Das Nachspiel eines unbekannten Autors war auf die Unterhaltung des Publikums orientiert (Vgl. ZAO, FVH, 163/93).

Die dramatische Tätigkeit Müllers in Rosswald war mit der Durchsetzung der Prinzipien verbunden, die für die Dramen der Aufklärung kennzeichnend waren. Dies demonstrierte sich nicht nur

12 Das Ballett wurde als Schlussveranstaltung zum Ritter-Trauerspiel ,Althestan, Herzog von Merzia“ aufgeführt, in dem Jungheim auch als Schauspieler tätig war (Vgl. SAU, G 3/ 010). 
in der Vorrede zu seinem Vorspiel, sondern auch in der vorgesehenen Einstudierung der Dramen Voltaires sowie im Vorspiel Müllers, Vereinigung des Trauerspiels, des Lustspiels, der Tonkunst und des Tanzes. ' Obwohl im avisierten Programm sowohl Dramen, als auch Ballett standen, verschmolzen sie nicht mehr zu einem Ganzen, wie es für die Aufführungen im Dienste des Barocks typisch war. Die Dramen- und Ballettauführungen waren bereits mit der thematischen sowie dramentheoretischen und choreographischen Autonomie verbunden.

Johann Heinrich Friedrich Müller hat 1761 das Rosswalder Herrschaftsgut verlassen. Er war kurz im Linzer Theater tätig, in dem er die Aufmerksamkeit des Grafen Franz Heinrich Schick erweckt hat. Auf Empfehlung dieses Adligen kam er in das Wiener Hoftheater, in dem er als Schauspieler engagiert wurde. (Vgl. Wurzbach 1856:382) In seinem dramatischen Schaffen überwiegen die Lustspiele. Dennoch ist der Beitrag seiner Tätigkeit nicht nur im künstlerischen Licht zu betrachten. Seine zum Teil biographisch geprägten Publikationen, Genaue Nachrichten von den beyden k. k. Schaubühnen und anderen öffentlichen Ergötzlichkeiten in Wien', ,Tagebuch von beyden k. k. Theatern in Wien' und ,J. H. F. Müller's Abschied von der k. k. Hof- und National-Schaubühne“ haben einen literaturhistorischen Wert, der unzählige Forschungsimpulse bietet.

\section{Schlussfolgerungen}

Das Rosswalder Schloss war bereits zu den Lebzeiten des Grafen Karl Joseph Hodiz (1673-1741) des Vaters des Grafen Albert Joseph Hoditz - mit der florierenden kulturellen Entfaltung verknüpft. Das kulturelle Engagement des Grafen Karl Joseph Hoditz stand im Dienste der barocken Repräsentation und im Rosswalder Schlosstheater wurden vor allem Opern der italienischen Provenienz aufgeführt.

Der Graf Albert Joseph Hoditz hat die kulturellen Aktivitäten seines Vaters nicht nur fortgesetzt, sondern auch beträchtlich entwickelt. Er hat den Rosswalder Schlossgarten zu einem Ort der künstlerischen Inspiration verwandelt. In dieser Schlossanlage befanden sich zahlreiche Statuen, die an die antike Mythologie erinnerten. Bereits durch diese Belebung der Antike wurde eine starke Verbindung zu Literatur und antiker Kultur erstellt, wodurch ein künstlerischer Hintergrund für die Dramen-, Opern- und Ballettaufführungen vorbereitet wurde. Diese Aufführungen fanden oft in der Gartenszenerie statt, die sich indirekt an der Mitgestaltung der Handlung beteiligte. In den Schlossgarten wurden beispielsweise bukolisch und anakreontisch geprägte Aufführungen situiert, denen diese „Naturbühne“ zweifelsohne mehr Authentizität verlieh.

Die im Rosswalder Schlosstheater aufgeführten Schauspiele wurden in den 1750er Jahren oft mit Musik, Gesang und Tanz verknüpft. Nicht selten fanden auf dieser Theaterbühne auch die sog. Hanswurstiaden statt, die meistens bereits überwunden wurden.

Zu einer allmähligen Verwandlung des Rosswalder Theaterrepertoires kam es Ende des Jahres 1757, in dem im Rosswalder Schloss Johann Heinrich Friedrich Müller angestellt wurde. Er verfügte über einen Einblick in die aktuelle Literaturentwicklung. Er formulierte zwölf Jahre vor dem Erscheinen der, Hamburgischen Dramaturgie‘ Gotthold Ephraim Lessings die Prinzipien, die für das aufklärerische Drama kennzeichnend waren. Zweifelsohne knüpfte er in seinen Reflexionen an die Aufführungen der Werke Lessings an. Er durfte seine Überlegungen jedoch auch an die Prinzipien des englischen elisabethanischen Theaters anlehnen, das vor allem durch die Werke William Shakespeares repräsentiert wurde. Im Rosswalder Schlosstheater wurde unter der Leitung Müllers lediglich fünf Jahre nach der Prämiere von Lessings, Miss Sara Sampson' dieses Trauerspiel aufgeführt. Müller konzentrierte sich jedoch nicht nur auf die deutschsprachige Literatur, sondern auch auf das literarische Schaffen anderer europäischer Autoren. In diesem Zusammenhang fand in Rosswald die Aufführung der Tragödie Voltairs ,Alziere‘ statt, in der bereits ausgewählte aufklärerische Dramenprinzipien umgesetzt wurden. Aus dem Vorwort zum Vorspiel Müllers geht hervor, dass dieser spätere berühmte Wiener Dramatiker große Aufmerksamkeit den schauspielerischen Leistungen geschenkt hat. Er hob vor allem die Rolle der ursprünglich von Aristoteles formulierten dramatischen Kategorien von Furcht und Leid hervor, die in die Katharsis münden. Diese Prinzipien wurden 
zur Zeit der Aufklärung wieder belebt, didaktisch untermauert und auf die Gesellschaftsschicht des Bürgertums bezogen.

Obwohl das Rosswalder Schloss an der Peripherie lag, wurde es zwischen 1750 und $1760 \mathrm{zu}$ einer friedevollen kulturellen Oase. Seine Kulturszene florierte in Übereinstimmung mit den neusten künstlerischen Tendenzen, die bei den Aufführungen im Schlosstheater sowie im Rosswalder Schlossgarten umgesetzt wurden.

\section{Literaturverzeichnis}

\section{Primärliteratur:}

Landesarchiv Troppau (ZAO), Achivalien des Großherrschaftsgut Groß Hoschütz (FVH): Vereinigung des Trauerspiels, des Lustspiels, der Tonkunst und des Tanzes, Inventarnr.: 163, Kartonnr.: 93.

Stattsarchiv Ulm (SAU), Theaterzettel zum Ballett „Der verjüngte Greis“ Johannes Jungheims, Sig.: G 3, Theaterzettel-Nr.: 010.

\section{Sekundärliteratur:}

Lessing, Gotthold Ephraim (1755): Miss Sara Sampson. Frankfurt an der Oder.

MüLLER, Johann Heinrich Friedrich (1770): Der Ball oder der versetzte Schmuck. Wien.

MüLLER, Johann Heinrich Friedrich (1770): Stirbt der Fuchs, so gilt's den Balg. Wien.

MüLLER, Johann Heinrich Friedrich (1771): Geschichte und Tagebuch der Wiener Schaubühne. Wien.

MüLlER, Johann Heinrich Friedrich (1771): Vier Narren in einer Person. Wien.

MÜLLER, Johann Heinrich Friedrich (1773): Genaue Nachrichten von den beyden k. $k$. Schaubühnen und anderen öffentlichen Ergötzlichkeiten in Wien. Wien.

MüLLER, Johann Heinrich Friedrich (1773): Die unähnlichen Brüder. Wien.

MüLlER, Johann Heinrich Friedrich (1775): Die Insel der Liebe, oder Amor, Erforscher der Herzen. Wien.

MüLLER, Johann Heinrich Friedrich (1802): J. H. F. Müllers Abschied von der k.k. Hof- und National-Schaubühne. Wien.

MYšKA, Milan (2011): Hrabě Hodic a jeho svět. Zámecká kultura ve Slezsku mezi barokem a osvícenstvim. Ostrava.

Tralles, Balthasar Ludwig (1776): Schattenriss der Annehmlichkeiten von Roswalde. Breslau.

WurzBACH, Constantin von (1856): Müller, Johann Heinrich Friedrich. In: Biographisches Lexikon des Kaiserthums Oesterreichenthaltend die Lebensskizzen der denkwürdigen Personen, welche 1750 bis $1850 \mathrm{im}$ Kaiserstaate und in seinen Kronländern gelebt haben. Bd. 19. Wien.

ZLÁ, Iveta (2019): Johann Heinrich Friedrich Müllers (1778-1815) Rosswalder dramatisches Intermezzo. In: Germanoslavica. Zeitschrift für germano-slawische Studien. Nr. 30(2), S. 87-99.

ZLÁ, Iveta (2020): Ausgewählte westschlesische Schlossresidenzen als kulturelle Repräsentanzen zwischen Barock und Aufklärung. Abriss der Problematik im literatur- und kulturhistorischen Blickwinkel. In: ACC journal. Nr. 2, S. 63-71.

\section{Internetquellen:}

URL 1: Geneanet. Zugänglich unter: https://en.geneanet.org/ [13.8.2021] 